Communications in Physics, Vol. 24, No. 3 (2014), pp. 193-200

DOI:10.15625/0868-3166/24/3/5027

\title{
MAGNETIC PROPERTIES OF QUANTUM SQUARE LATTICE HEISENBERG ANTIFERROMAGNET WITH FRUSTRATION: AN AUXILIARY FERMION APPROACH
}

\author{
PHAM THI THANH NGA \\ Water Resources University, 175 Tay Son, Hanoi \\ NGUYEN TOAN THANG \\ Institute of Physics, Vietnam Academy of Science and Technology \\ E-mail: nga_ptt@wru.edu.vn \\ Received 17 May 2014 \\ Accepted for publication 22 July 2014
}

\begin{abstract}
We study the magnetic ordered phases of the spin-1/2 Heisenberg antiferromagnetic model with nearest $J_{1}$ and next nearest neighbor $J_{2}$ exchange interactions on the square lattice using fermionic representation of spin operators within Popov-Fedotov approach.The unphysical states are eliminated on each site by introducing an imaginary chemical potential. Working in local coordinate system for every site we investigate the different ordered phases in dependence on exchange parameters. At $T=0$ we recover the conventional spin wave results.

Keywords: functional integral, antiferromagnetic Heisenberg model, square lattice, frustration.
\end{abstract}

\section{INTRODUCTION}

Recently, geometric frustration in spin systems has attracted a lot of interest. The discovery of the ceramic high-temperature superconductivity has stimulated great interests in studying possible novel phases in frustrated quantum antiferromagnet on two-dimensional lattices [1]. The square lattice antiferromagnetic Heisenberg model with frustration introduced by next nearest neighbor exchange interaction has been investigated by many authors with various approximate and numerical methods: the spin wave theory [2,3]; small lattice calculations [4]; the Schwinger boson theory [5]; series expansion [6] and other methods [7].

However, there is a considerable discrepancy between the results of above works, in particular for the ground state properties of the system. The reason is the complex nature of spin operators.

The non - canonical commutation relations of spin operators pose a severe difficulty for analytical treating quantum spin systems. Because spin operators satisfy neither Fermi nor Bose commutation relations, there is no Wick theorem directly for spin operators. Therefore, the standard methods widely used for pure Bose or Fermi systems cannot be applied. Several approaches have been developed for the investigation of spin systems. One can formulate a coupled system of (C)2014 Vietnam Academy of Science and Technology 
Heisenberg equations for the spin Green functions. In order to solve the coupled system of Green functions one has to decouple the higher order functions by some plausible criterion. As a result, one cannot systematically estimate the decoupling error. The second method is based on various representation of spin operators by some auxiliary fields such as Bose operators (Schwinger bosons, Holstein-Primakoff or Dyson-Maleev representation) or Fermi operators. However, in all cases, the representation of spin operators as a bilinear combination of canonical operators enlarges the dimension of the Hilbert space of these auxiliary fields, so the spurious unphysical states appear and should be eliminated from the consideration.

The exclusion of these unphysical states may be done by some local constraint procedure with the fixed number of auxiliary particle on each site. Unfortunately, on the most cases, one cannot treat the local constraint exactly and usually one replaces the local constraint by a global one, where the number of auxiliary particles is fixed only as an average number for all sites. It is known that this replacement is questionable because the approximation for spins became uncontrollable. In order to avoid the main difficulty related with the local constraint in 1988 Popov and Fedotov suggested a new simple method, where the spin $1 / 2$ and spin 1 operators are represented by the fermions with imaginary chemical potential [8]. This allows the application of standard fermionic perturbation techniques for spin systems. Later, the Popov-Fedotov trick was extended to arbitrary spin by introducing propre chemical potential for auxiliary fermions.

Recently, the Popov-Fedotov method was generalized to boson and fermion models with various constraints on Fock states. This extension leads to the possible successful application of Bold Diagrammatic Monte Carlo to quantum spin systems [9]. While the paper [8] are basically of a methodological nature, the Popov-Fedotov approach has been used for investigation of ordered phase different particular spin systems: the three dimensional ferromagnetic Heisenberg model [10], the isotropic Heisenberg antiferromagnets on a square, hypercubical $[11,13]$ and triangular lattices [14]. The Popov-Fedotov has been applied successfully also to the negative-U Hubbard Model [15], spin glass model [16]...

In this paper we apply the Popov-Fedotov trick to a square lattice frustrated Heisenberg antiferromagnet. In what follows in Sec. II we introduce the model and its fermionization. In Sec. III we derive the free energy and the sublattice magnetization in the mean field and the one loop approximations. The last section is devoted to discussions.

\section{THE MODEL AND ITS FERMIONIZATION}

We consider the frustrated square lattice Heisenberg model defined by the Hamiltonian:

$$
H=J_{1} \sum_{<i j>} \mathbf{S}_{i} \mathbf{S}_{j}+J_{2} \sum_{<<i k>>} \mathbf{S}_{i} \mathbf{S}_{k}
$$

where $J_{1}, J_{2}>0$ describe the exchange interaction between the nearest neighbors and the nextnearest neighbors on the square lattice of $N$ sites. The operators $\mathbf{S}_{i}$ are the quantum spin operators on lattice site $i$. We study here the extreme quantum case $S=1 / 2$, when quantum fluctuations tend to be the largest. For the classical limit $(S=\infty)$, the model has a phase transition at $J_{2} / J_{1}=1 / 2$, below which the ground state has the conventional Neel order with two sublattices. For large value of the coupling $J_{2}$, which introduces frustrations into the model the Neel state cannot be stable and in the large $J_{2}$ limit the above mentioned sublattices are decoupled and each of them has its 
own antiferromagnetic order. In this paper we consider the Neel state (AF) with the ordering wave vector $\mathrm{Q}=(\pi, \pi)$ and the so-called collinear phase (AFC) with $\mathrm{Q}=(\pi, 0)$.

In our previous work [14] we have shown that the magnetic order state of spin $1 / 2$ antiferromagnetic Heisenberg model with nearest-neighbors coupling on hypercubic and triangular lattices may be studied in rather simple unified way using the Popov-Fedotov trick in combination with a transformation to the local coordinates defined for every site. The extension to the model (1) with the next-nearest-neighbors bond $J_{2}$ is straightforward. We adopt the same notations as in Ref [14]. Firstly, we rewrite the Hamiltonian (1) in the new local spatially varying coordinates Ox'y'z' with Oz' pointing along the local classical Neel direction, the y'-axis is invariable:

$$
H=J_{1} \sum_{<i j>}\left\{S_{i}^{y} S_{j}^{y}-\left(S_{i}^{x} S_{j}^{x}+S_{i}^{z} S_{j}^{z}\right)\right\}+J_{2} \sum_{<<i k>>} \mathbf{S}_{i} \mathbf{S}_{k}
$$

Then we represent the spin operators in term of auxiliary Fermi operators:

$$
S_{i}^{\alpha}=\frac{1}{2} \sum_{\sigma \sigma^{\prime}} a_{i \sigma}^{+} \tau_{\sigma \sigma^{\prime}}^{\alpha} a_{i \sigma^{\prime}}
$$

where $\tau=\left(\tau^{x}, \tau^{y}, \tau^{z}\right)$ are the Pauli matrices. Because the Fock state of the fermion $a_{i \sigma}$ is spanned by four states, the two unphysics states have to be excluded by the constraint:

$$
\widehat{N}_{i}=\sum_{\sigma} a_{i \sigma}^{+} a_{i \sigma}=1
$$

As it was shown by Popov-Fedotov, the constraint is enforced by introducing to the partition function the projection operators:

$$
P=\frac{1}{i^{N}} e^{i \frac{\pi}{2} \hat{N}}
$$

where $\hat{N}=\sum_{i} \hat{N}_{i}$. As a result, the fermionic Matsubara frequencies are modified as follows:

$$
\tilde{\omega}_{F}=\omega_{F}-\frac{\pi}{2 \beta}=\frac{2 \pi}{\beta}\left(n+\frac{1}{4}\right)
$$

\section{MEAN FIELD AND ONE LOOP EQUATIONS}

The 4-fermion terms in the partition function may be eliminated by a Hubbard-Stratonovich transformation, introducing the Bose auxiliary field $\varphi_{i}$. After integration over the bilinear Grassmann variables, the partition function of the Hamilton (1) may be written as follows:

$$
Z=\frac{1}{i^{N}} \frac{1}{Z_{o}} \int_{\varphi(B)=\varphi(o)}[D \varphi] e^{-S_{e f f}[\varphi]}
$$

with the effective action $S_{e f f}[\varphi]$ being:

$$
S_{e f f}[\varphi]=\int_{0}^{\beta} S_{o}[\varphi] d \tau-\ln \operatorname{det} \beta \hat{K}
$$


At the one loop approximation the effective action on Eq. (8) is given by:

$$
\begin{aligned}
S_{e f f}= & \frac{\beta}{2} \sum_{\Omega_{n}} \bar{\Phi}_{i o} \hat{A}_{i j}\left(\Omega_{n}\right) \Phi_{\mathrm{io}}-\sum_{\mathrm{i}} \ln \frac{2}{\mathrm{i}} \cosh \frac{\beta\left|\varphi_{\mathrm{io}}\right|}{2} \\
& +\frac{\beta}{2} \sum_{i j, \Omega_{n}}\left\{\bar{\Phi}_{i o} \hat{C}_{i j}\left(\Omega_{n}\right) \delta \Phi_{\mathrm{j}}\left(\Omega_{n}\right)+\delta \bar{\Phi}_{\mathrm{i}}\left(-\Omega_{n}\right) \hat{C}_{i j}\left(\Omega_{n}\right) \Phi_{\mathrm{jo}}\right\} \\
& +\frac{\beta}{2} \sum_{i j, \Omega_{n}}\left\{\delta \bar{\Phi}_{\mathrm{i}}\left(-\Omega_{n}\right) \hat{D}_{i j}\left(\Omega_{n}\right) \delta \Phi_{\mathrm{j}}\left(\Omega_{n}\right)\right\}
\end{aligned}
$$

where the following notations are used:

$$
\begin{aligned}
& \hat{A}_{i j}\left(\Omega_{n}\right)=\delta_{\Omega_{n}, 0}\left(\hat{J}^{-1}\right)_{i j} \\
& \hat{C}_{i j}\left(\Omega_{n}\right)=\delta_{\Omega_{n}, 0}\left(\hat{J}^{-1}\right)_{i j}+\frac{2}{\beta} \hat{K}_{i 1} \delta_{i j} \delta_{\Omega_{n}, 0} \\
& \hat{D}_{i j}\left(\Omega_{n}\right)=\delta_{\Omega_{n}, 0}\left(\hat{J}^{-1}\right)_{i j}+\frac{1}{\beta} \hat{K}_{i 2} \delta_{i j} \delta_{\Omega_{n}, 0}
\end{aligned}
$$

The three component spinors in terms of the components of the auxiliary field $\varphi_{i}$ are introduced. $\left(\hat{J}^{-1}\right)_{i j}$ is the inverse matrix of the exchange coupling. $\hat{K}_{i 1}$ is given by:

$$
\hat{K}_{i 1}=-\frac{1}{4} \frac{\beta}{\left|\varphi_{\text {io }}\right|} \tanh \frac{\beta\left|\varphi_{\text {io }}\right|}{2} \hat{I}
$$

Because we are working in the local coordinate systems then in mean field level we have: $\varphi_{i o}^{ \pm}=0$; $\varphi_{i o}^{z}=\varphi_{o}$ for every site $i$ and $K_{i 2}^{\alpha \beta}$ become:

$$
\begin{aligned}
K_{i 2}^{+-} & =\left(K_{i 2}^{-+}\right)^{*}=-\frac{\beta}{4} \frac{1}{\left(\varphi_{\mathrm{io}}+\mathrm{i} \Omega\right)} \tanh \frac{\beta \varphi_{\mathrm{o}}}{2} \\
K_{2}^{z z} & =-\frac{\beta}{4}\left(1-4 m_{o}^{2}\right) \delta_{\omega, 0}
\end{aligned}
$$

Following the least action principle, the mean field equation for the auxiliary field $\varphi_{i o}$ is written as:

$$
\sum_{j}\left(\hat{J}^{-1}\right)_{i j} \Phi_{\mathrm{jo}}=\frac{\Phi_{\mathrm{io}}}{2\left|\varphi_{\mathrm{io}}\right|} \tanh \frac{\beta\left|\varphi_{\mathrm{io}}\right|}{2}
$$

The sublattice magnetization is related to the averaged of the auxiliary field $\left\langle\varphi_{j}\right\rangle$ as:

$$
m_{i}^{\alpha}=\sum_{j, \beta}\left(J^{-1}\right)_{i j}^{\alpha \beta} \varphi_{j}^{\beta}
$$

From Eq. (16) and Eq. (17) we obtain the mean field equation of the magnetization as follows:

$$
m_{i o}=\frac{1}{2} \tanh \frac{\beta \sum_{j}(\hat{J})_{i j}^{z z} m_{j o}}{2}
$$

In the absence of the external magnetic field all the sublattices are equivalent and from Eq. (18) one gets:

$$
m_{o}=\frac{1}{2} \tanh \left[2 \beta\left(J_{1}-J_{2}\right) m_{o}\right]
$$


for AF state and:

$$
m_{o}=\frac{1}{2} \tanh \left(2 \beta J_{2} m_{o}\right)
$$

for AFC state. The above equations lead to the phase transition temperature $T_{C}$ :

$$
k_{B} T_{C}=\left(J_{1}-J_{2}\right)
$$

for AF state and:

$$
k_{B} T_{C}=J_{2}
$$

for AFC state. For both phases the critical temperatures are twice larger than the Neel temperatures obtained in the case of using global constraint instead of local one. In order to take into account the fluctuation correction we perform the Fourier transformation for the last term of the affective action in Eq. (9) and obtain:

$$
S_{e f f}^{(2)}=\frac{\beta}{2} \sum_{p, \Omega} \delta \bar{\Phi}(-p,-\Omega) \hat{D}(p, \Omega) \delta \Phi(p, \Omega)
$$

where the kernel $\hat{D}(p, \Omega)$ reads:

$$
\hat{D}(p, \Omega)=\tilde{J}^{-1}(p)+\frac{1}{\beta} \hat{K}_{2}(p)
$$

On Eq. (24) we use the following notations:

$$
\hat{K}_{2}(p)=\left(\begin{array}{ccc}
0 & K_{2}^{+-} & 0 \\
K_{2}^{-+} & 0 & 0 \\
0 & 0 & K_{2}^{z z}
\end{array}\right)
$$

with:

$$
K_{i 2}^{+-}=\left(K_{i 2}^{-+}\right)^{*}=-\frac{m_{o}}{2\left(4 J m_{o}+i \Omega\right)}
$$

and $K_{2}^{z z}$ is given by Eq. (15). $\tilde{J}^{-1}(p)$ is the Fourier transformation of the coupling $\left(J^{-1}\right)_{i j}^{\alpha \beta}$ in the $(+,-, z)$ basics. Intergrating over the fluctuation field $\delta \Phi(\Omega)$ we obtain the fluctuation contribution to the partition function:

$$
Z_{f l}=\left[\operatorname{det}\left(\hat{I}+\tilde{J}(p) \hat{K}_{2}(p)\right)\right]^{1 / 2}
$$

From Eq. (2) we get the following non-zero components of the coupling matrix $J(p)$ in $(x, y, z)$ basics. For AF phase:

$$
\begin{aligned}
& J^{x x}(p)=J^{z z}(p) \equiv X=4 J_{1}[\gamma(p)-\alpha \eta(p)] \\
& J^{y y}(p) \equiv Y=-4 J_{1}[\gamma(p)+\alpha \eta(p)]
\end{aligned}
$$

For AFC phase:

$$
\begin{aligned}
& X=J^{x x}(p)=J^{z z}(p) \equiv X=2 J_{1}\left[2 \alpha \eta(p)+\cos p_{x}-\cos p_{y}\right] \\
& Y=J^{y y}(p)=-4 J_{1}[\gamma(p)+\alpha \eta(p)]
\end{aligned}
$$


where:

$$
\begin{aligned}
\gamma(p) & =\frac{1}{2}\left(\cos p_{x}+\cos p_{y}\right) \\
\eta(p) & =\cos p_{x} \cdot \cos p_{y} \\
\alpha & =J_{2} / J_{1} .
\end{aligned}
$$

Then in the basics $(+,-, z)$, the coupling matrix $\tilde{J}$ is determined by:

$$
\tilde{J}=\left(\begin{array}{ccc}
X-Y & X+Y & 0 \\
X+Y & X-Y & 0 \\
0 & 0 & X
\end{array}\right)
$$

Using Eq. (15), Eq. (26) and Eq. (31) we arrive at:

$$
\operatorname{det}\left(\hat{I}+\tilde{J}(p) \hat{K}_{2}(p)\right)=\prod_{p}\left(1+X K_{2}^{z z}\right) \prod_{\Omega} Q(\Omega)
$$

where:

$$
Q=1+(X+Y)\left(K_{2}^{-+}+K_{2}^{+-}\right)+4 X Y K_{2}^{-+} K_{2}^{+-}
$$

The product over the bosonic Matsubara frequencies may be performed using the following relation:

$$
\prod_{n=0}^{\infty}\left[\left(1+\frac{a}{(n+b)}\right)\left(1-\frac{a}{n+c}\right)\right]=\frac{\Gamma(b) \Gamma(c)}{\Gamma(a+b) \Gamma(c-a)}
$$

with $\Gamma(z)$ being the Gamma function. Then the free energy in the one loop approximation is given by:

$$
\begin{aligned}
F= & \frac{N a m_{o}^{2}}{2}-\frac{N}{\beta} \ln \left(2 \cosh \frac{\beta a m_{o}}{2}\right)+\frac{1}{\beta} \sum_{p \in B Z} \ln \frac{\sinh \frac{\beta a m_{o} \omega(p)}{2}}{\sinh \frac{\beta a m_{o}}{2}} \\
& +\frac{1}{2 \beta} \sum_{p \in B Z} \ln \left[1-\frac{\beta}{4}\left(1-4 m_{o}^{2}\right) X\right]
\end{aligned}
$$

where:

$$
\omega^{2}(p)=\left(1-\frac{X}{a}\right)\left(1-\frac{Y}{a}\right)
$$

and:

$$
m_{o}=\frac{1}{2} \tanh \frac{\beta a m_{o}}{2}
$$

For AF phase $a=4 J(1-\alpha)$ and $X, Y$ are given by Eq. (28). For AFC phase $a=4 \alpha J$ and $X, Y$ are given by Eq. (29). We note here that the derivation of the free energy Eq. (35) in this paper is carried out by different way in comparison with the one in [14] and seems to be much more simpler.

In order to calculate the sublattice magnetization in one loop approximation, we introduce local field $\vec{B}_{i}$ in the direction of the local classical magnetization. The free energy in the presence of the local magnetic field $F(B)$ is given by the same Eq. (35) with the following substitutions:

$$
\begin{aligned}
& m_{o} \rightarrow m_{o}+\Delta m B \\
& \Delta m=\frac{\frac{\beta}{2}\left(1-4 m_{o}^{2}\right)}{1+\frac{\beta a}{4}\left(1-4 m_{o}^{2}\right)}
\end{aligned}
$$


The sublattice magnetization is given as follows:

$$
m=\left[-\frac{\partial F(B)}{\partial B}\right]_{B=0}
$$

From (35), (38), and (39) we obtain:

$$
\begin{aligned}
& m(\mathrm{~T})=m_{o}+\frac{1}{4 m_{o}}(1+a \Delta m)-\frac{m_{o} \Delta m}{N} \sum_{p \in B Z} \frac{X}{1-\frac{\beta X}{4}\left(1-4 m_{o}^{2}\right)} \\
& -\frac{1}{4 N} \sum_{p \in B Z} \operatorname{coth} \frac{\beta a m_{o} \omega(p)}{2}\left[\frac{2}{\omega(p)}-\frac{X+Y}{a \omega(p)}+2 a \Delta m \omega(p)\right]
\end{aligned}
$$

\section{DISCUSSIONS}

The Eq. (35), Eq. (36) and Eq. (40) are the main analytical result of the paper, where the free energy $F$, the elementary excitation $\omega(p)$ and the sublattices magnetizations are derived for both magnetically ordered phases AF and AFC. Putting $\alpha=0$ we recover the results of Refs. [11-13] for the unfrustrated fermionized Heisenberg model in a square lattice. Similar to the unfrustrated case the results obtained by Popov-Fedotov procedure in the one loop approximation for both AF and AFC phases lead in the limit of zero temperature exactly to the same ones derived for the next to leading term in the linear spin wave theory $[2,3]$. We numerically calculated the ground state energies and the magnetizations at $T=0$ for both phases. At $\alpha=0.5008$ the Neel state energy become larger than the ground state of collinear phase. At $\alpha=0.384$, the Neel sublattice magnetization changes its sign, while at $\alpha=0.495$ the collinear magnetization equals to zero. It means that in a finite region of the frustration parameter $a$, the ordered phases are destabilized due to quantum fluctuations. It is essentially the same result of linear spin wave theory [2]. It is not surprising, because at one loop approximation, taking into account the local constraint does not improve zero temperature result obtained by treating the constraint in global approximation [11, 13]. At finite temperatures the Popov-Fedotov approach estimate better the thermal fluctuations [9] and it would change the result considerably. We expect that the region of disordered phase would be notably enlarged due to fluctuations even at rather low temperatures. In order to get better result at $T=0$, we have to either go over the one loop approximation or to modify the Popov-Fedotov method [10]. It is left for future.

\section{ACKNOWLEDGMENT}

This work is funded by the Vietnam National Foundation for Science and Technology Development (NAFOSTED) under Grant No.103.02 - 2011.5.

\section{REFERENCES}

[1] J. Richter, Schalenburg and A. Honecker, Quantum magnetism in two-dimensional disorder in quantum magnetism, U Scholwock (Ed.) Lecture notes in Physics, Springer 645 (2004) 85.

[2] P. Chandra and Doucot, Phys. Rev. B 38 (1988) 9335.

[3] A. V. Dotsenko and O. P. Sushkov, Phys. Rev B50, (1994) 13821.

[4] N. B. Ivanov and Richter, J. Phys. Condens. Matter 6, (1994) 3785.

[5] F. Mila, D. Poilblanc and C. Bruder, Phys. Rev. B 43, (1991) 7891.

[6] M. P. Gelfand, Phys. Rev. B 42, (1990) 8206.

[7] A. Yamada, K. Seki, R. Eder, Y. Ohta, Phys. Rev. B 88, (2013) 075114.

[8] V. N. Popov and S. A. Fedotov, Sov. Phys. JETP, 67(1988) 535. 
[9] J. Brinckmannand P. Wolfle, arXiv.cond-mat. 08033312.

[10] S. Tejimaand A. Oguchi, J. Phys. Soc. Jpn 64, (1995)4923.

[11] S. Azakov et al,Int. J. Mod. Phys. B 14, (2000) 13.

[12] R. Dillenschneider and J. Richert, Eur. Phys. J. B 49, (2006) 187.

[13] R. Dillenschneider and J. Richert, Phys. Rev. B 73, (2006) 2440.

[14] Pham Thi Thanh Nga, Nguyen Toan Thang, Communications in Physics 22(1) (2012) 33, Comm. in Phys. 22(4) (2012) 383 Erratum.

[15] J. Stein and R. Oppermann, Phys. Rev. B 46, (1992) 8409.

[16] R. Oppermann and A. Muller-Groeling, Phys. Lett. A 168 (1992) 75. 